\title{
Orthogonal experiment analysis of Carbon nanotubes oxidation process
}

\author{
Hongwei $\mathrm{LU}^{\mathrm{a}}$, Liming ZOU ${ }^{\mathrm{b}}$, Yizhe $\mathrm{WEI}^{\mathrm{c}}$ and Yongjing $X \mathrm{U}^{\mathrm{d}}$
}

State Key Laboratory for Modification of Chemical Fibers and Polymer Materials,

College of Materials Science and Engineering, Donghua University, 2999 North

People Road, Shanghai 201620, People's Republic of China

aemail: luhw0801@sina.com, bemail: Imzou@dhu.edu.cn,

cemail: In_2008@163.com, ${ }^{\mathrm{d} e m a i l}:$ kittyxyj@163.com

Keywords: Carbon nanotubes; Oxidation; Orthogonal experiment analysis

\begin{abstract}
The application of carbon nanotubes (CNTs) in biomaterials field was limited by its poor dispersion in water solution. In this paper, a specific methodology based on potassium permanganate $\left(\mathrm{KMnO}_{4}\right)$ /concentrated sulfuric acid $\left(\mathrm{H}_{2} \mathrm{SO}_{4}\right)$ hydrothermal oxidation was used to modify the surface of multi-walled carbon nanotubes (MWCNTs). The orthogonal experiment results indicated that $\mathrm{MWCNTs} / \mathrm{KMnO}_{4} /$ concentrated $\mathrm{H}_{2} \mathrm{SO}_{4}$ at 1:3:100 (g/g/g), stirring under $150^{\circ} \mathrm{C}$ for $7 \mathrm{~h}$ was the optimal oxidation process. FT-IR and TGA results demonstrated that carboxyl groups were introduced to the surface of MWCNTs. Raman spectra and SEM, TEM showed that structure of MWCNTs were damaged after been oxidized.
\end{abstract}

\section{Introduction}

Carbon nanotubes (CNTs) hold promise as one of the key nanomaterials since it was discovered by Iijima in 1991[1]. CNTs have attracted much interest due to their unique properties such us excellent mechanical strength, high electrical conductivity, chemical stability [2] and CNTs have been widely applied in composite [3], hydrogen storage materials[4], field emission devices[5], biomaterials[6] and other fields.

The preparation of promising nanomaterials based on CNTs requires high dispersibility of CNTs in solvent or base materials. CNTs are easily aggregated in water solution for the predominantly hydrophobic nature of carbon materials. Covalent modification is an efficient method to improve the dispersion of CNTs in solvents. Typical hydrothermal oxidation method are based on sulphuric acid $\left(\mathrm{H}_{2} \mathrm{SO}_{4}\right)$, nitric acid $\left(\mathrm{HNO}_{3}\right), \mathrm{HNO}_{3}-\mathrm{H}_{2} \mathrm{SO}_{4}$ mixtures or potassium permanganate $\left(\mathrm{KMnO}_{4}\right)$ [7].

In this paper, a specific methodology based on potassium permanganate $\left(\mathrm{KMnO}_{4}\right) /$ concentrated sulfuric acid $\left(\mathrm{H}_{2} \mathrm{SO}_{4}\right)$ hydrothermal oxidation was used to modify MWCNTs. Orthogonal experiment were taken to study the process. The as prepared functional MWCNTs show excellent dispersion in water. This work can offer us a systematic method to prepare functional CNTs and realized its application in many fields.

\section{Materials and method}

Pristine MWCNTs were purchased from Shenzhen Nanotech Port Co., Ltd with purity of 90\%, lengths $>1.5 \mu \mathrm{m}$ and diameters $<10 \mathrm{~nm}$. $\mathrm{H}_{2} \mathrm{SO}_{4} \mathrm{KMnO} 4$ were purchased from Sinopharm ChemicalReagent Co., Ltd. (China) and used as received. $\mathrm{HCl}$ and $\mathrm{H} 2 \mathrm{SO} 4$ were purchased from Shanghai daruifine-chemical Co., Ltd (China).

Fourier transform infrared spectral (FT-IR) in the range of $400-4000 \mathrm{~cm}-1$ were obtained by a Nicolet 8700 Fourier transform infrared spectrometer (thermo Electron, American) with KBr pellets of MWCNTs, and under the averaging of 32 scans at a resolution of $4 \mathrm{~cm}-1$. Thermogravimetric analyses (TGA) were performed by heating the sample under nitrogen flow from $30^{\circ} \mathrm{C}$ to $900^{\circ} \mathrm{C}$ at $20 \mathrm{~K} / \mathrm{min}$ using a TG 209 F1 Iris thermogravimetric analyzer (Netzsch-GeraetebauGmbH, 
Germany). The Raman spectra were performed using a Renishaw inVia-Reflex spectrometer (UK) with excitation line set to $\lambda=633 \mathrm{~nm}$, and the measurements were recorded from $600 \mathrm{~cm}-1$ to 4000 cm-1 on each MWCNTs sample. Scanning electron microscopy (SEM) was carried out on a JEOL JSM-5600 LV instrument (Japan) operating at 5KV. Transmission electron microscopy (TEM) was taken using a NEC2100F transmission electron microscopy (Japan).

MWCNTs have been purified before we used [8]. $10 \mathrm{~g}$ concentrated H2SO4 (98\%) were diluted to $0.5 \mathrm{~mol} / \mathrm{L}$ and added to a $1000 \mathrm{ml}$ round-bottom flask, then $100 \mathrm{mg}$ MWCNTs and $300 \mathrm{mg}$ $\mathrm{KMnO} 4$ followed. The mixture were treated under a refluxed condenser for $7 \mathrm{~h}$ at $150^{\circ} \mathrm{C}$. After that, $10 \mathrm{ml} \mathrm{HCl}(37 \%)$ were added to the mixture to neutralize superfluous $\mathrm{KMnO}_{4}$. Then the product was filtrated and washed by water until neutral. Finally, the functional MWCNTs were dried in a vacuum oven at $75^{\circ} \mathrm{C}$ over night.

\section{Result and Discussion}

\section{Orthogonal experiment analysis}

In this paper, $\mathrm{H}_{2} \mathrm{SO}_{4} / \mathrm{KMnO}_{4}$ were used as oxidant. Ratio of $\mathrm{H}_{2} \mathrm{SO}_{4} / \mathrm{KMnO}_{4}$ influence the oxidability of $\mathrm{KMnO}_{4}$. Ratio of $\mathrm{MWCNTs} / \mathrm{KMnO}_{4}$, reaction temperature, reaction time influence the oxidation results of MWCNTs. So the effect factors of oxidation process were designed as follows.

Table1. Effect factors of MWCNTs oxidation process

\begin{tabular}{ccccc}
\hline & $\mathrm{A}$ & $\mathrm{B}$ & $\mathrm{C}$ & $\mathrm{D}$ \\
& $\begin{array}{c}\mathrm{H}_{2} \mathrm{SO}_{4} / \mathrm{KMnO}_{4} \\
(\mathrm{~g} / \mathrm{mg})\end{array}$ & $\begin{array}{c}\text { MWCNTs/KMnO} \\
(\mathrm{g} / \mathrm{g})\end{array}$ & $\begin{array}{c}\text { Temperature } \\
\left({ }^{\circ} \mathrm{C}\right)\end{array}$ & $\begin{array}{c}\text { Time } \\
(\mathrm{h})\end{array}$ \\
\hline 1 & $10 / 200$ & $1 / 1$ & 100 & 7 \\
2 & $10 / 300$ & $1 / 2$ & 125 & 9 \\
3 & $10 / 400$ & $1 / 3$ & 150 & 11 \\
\hline
\end{tabular}

According to Table1 we designed a series of orthogonal experiment as shown in Tabl2.

Table2. Design of orthogonal experiment

\begin{tabular}{cllll}
\hline Experiment Number & A & B & C & D \\
\hline 1 & A1 & B1 & C1 & D1 \\
2 & A1 & B2 & C2 & D2 \\
3 & A1 & B3 & C3 & D3 \\
4 & A2 & B1 & C2 & D3 \\
5 & A2 & B2 & C3 & D1 \\
6 & A2 & B3 & C1 & D2 \\
7 & A3 & B1 & C3 & D2 \\
8 & A3 & B2 & C1 & D3 \\
9 & A3 & B3 & C2 & D1 \\
\hline
\end{tabular}

According to Table2 we prepared a series of functional MWCNTs, Boehm titration were used to detect the content of carboxyl groups on the surface of MWCNTs and evaluate the effect of oxidation process. The results were presented in Table3 and the result analyses were displayed in Table4. 
Table3. Content of carboxyl groups on the surface of MWCNTs

\begin{tabular}{cc}
\hline Experiment Number & content of carboxyl groups (mmoL/g) \\
\hline 1 & 2.68 \\
2 & 2.44 \\
3 & 1.94 \\
4 & 2.94 \\
5 & 4.67 \\
6 & 2.86 \\
7 & 2.42 \\
8 & 1.56 \\
9 & 1.30 \\
\hline
\end{tabular}

Table4. Orthogonal experiment analysis result of content of carboxyl groups

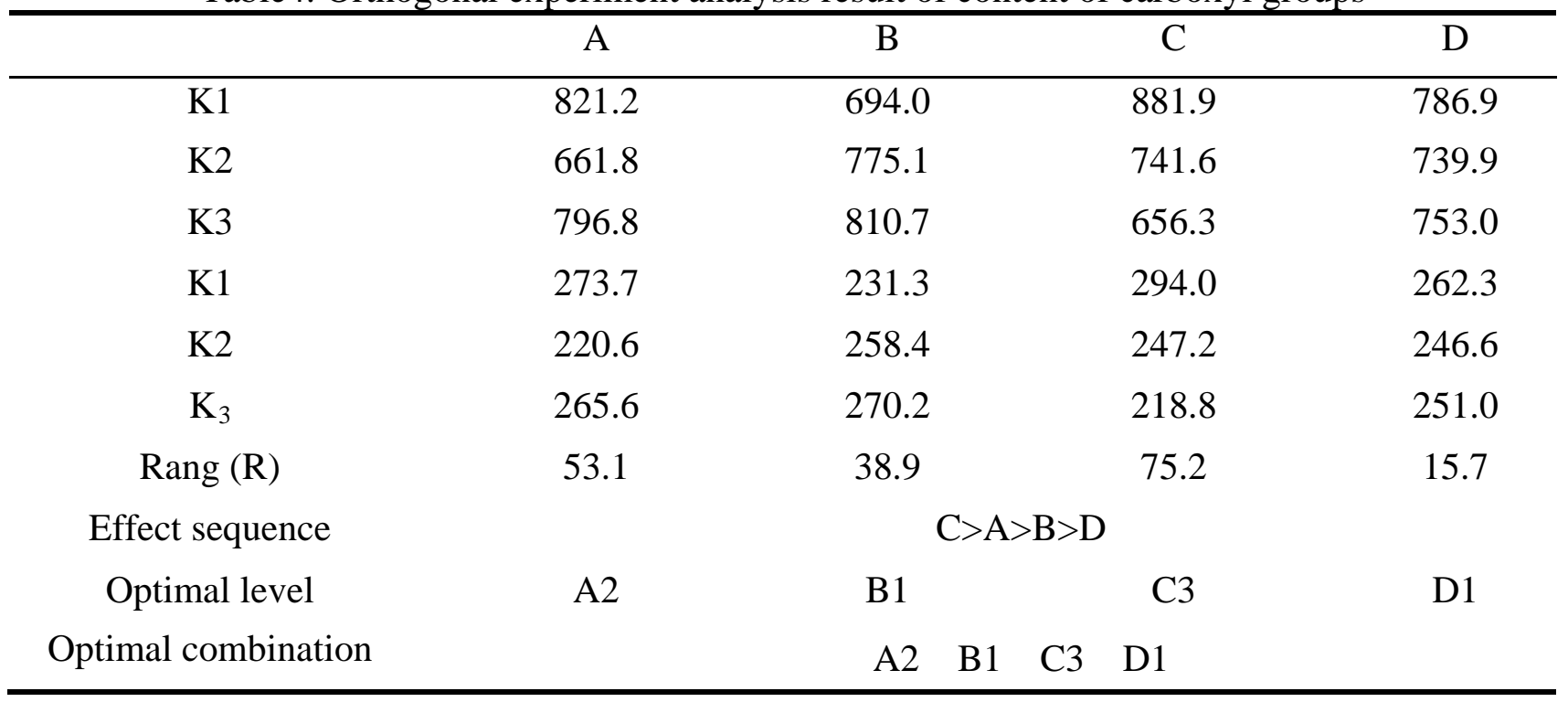

From the Orthogonal experiment analysis result, we got the optimal combination A2-B1-C3-D1, which means that at a $\mathrm{H}_{2} \mathrm{SO}_{4} / \mathrm{KMnO}_{4}$ (g/mg) $1: 300$, MWCNTs/KMnO 4 (g/g) 1:3, reaction temperature $150^{\circ} \mathrm{C}$, reaction time $7 \mathrm{~h}$ MWCNTs got the best oxidation effect.

\section{Characterization}

The FT-IR spectra were taken to characterize the functional groups on the surface of MWCNTs as showed in Figure 1. After modification O-MWCNTs shows a peak at $1740 \mathrm{~cm}^{-1}$ which is the stretching vibration for $\mathrm{C}=\mathrm{O}$, it means that MWCNTs has been modified and carboxyl groups were introduced to the surface of MWCNTs. In addition, obvious $\mathrm{CH}_{3}$ bending vibration peak and C-O stretching vibration peak appear at $1380 \mathrm{~cm}^{-1}$ and $1000 \sim 1100 \mathrm{~cm}^{-1}$, it means that some other kind of oxygen containing groups were also introduced and the surface defect increased.

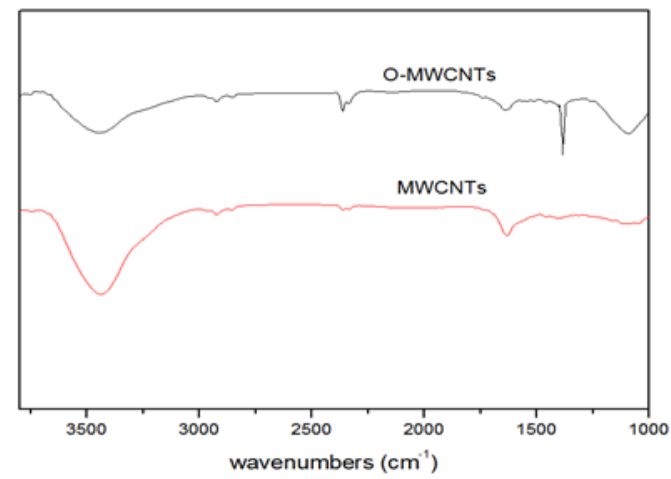

Figure 1. FT-IR spectra of MWCNTs and O-MWCNTs

TGA is a practical thermal analysis technique that can be used to evaluate the thermal stability and content of a materials. Figure 2 showed the TGA curves of MWCNTs and O-MWCNTs at a 
temperature range from $30-500^{\circ} \mathrm{C}$. O-MWCNTs have a significant mass loss at $200-500^{\circ} \mathrm{C}$, which is the functional groups decompose temperature range. It can be used to prove that functional groups have been introduced to the surface of MWCNTs after been modification.

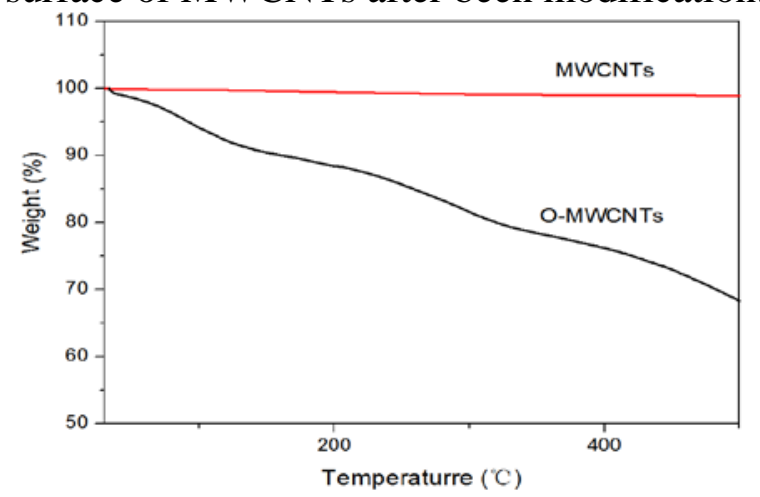

Figure 2. TGA curves of MWCNTs and O-MWCNTs

The morphology of MWCNTs and O-MWCNTs were observed using SEM and TEM (Figure 3). As can be seen from Figure 3a and 3b, MWCNTs aggregated together while O-MWCNTs homogeneous dispersed due to the functional groups introduced to the surface of O-MWCNTs. Figure 3c and 3d demonstrated that after modification, diameter of MWCNTs decreased and the port of tubes were opened, it proves that the structure of O-MWCNTs were damaged.
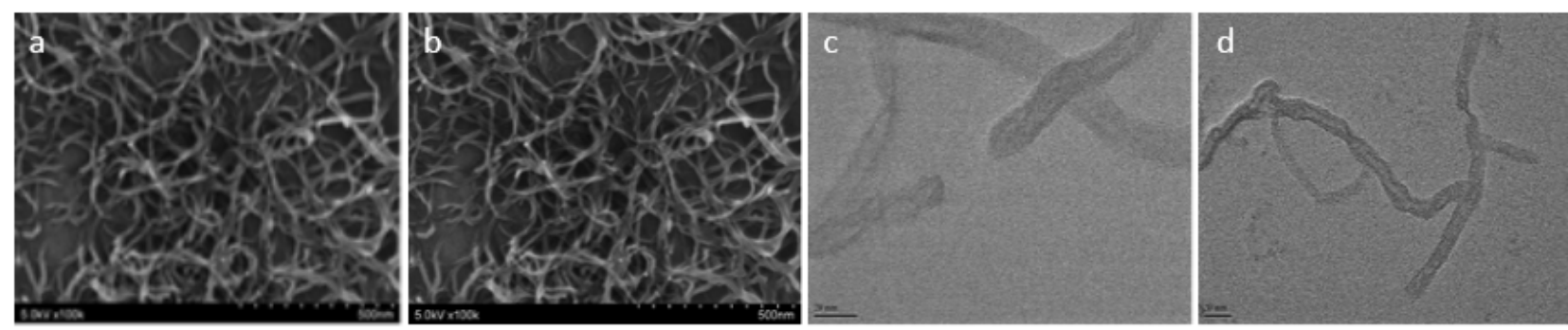

Figure 3. SEM (a, b) and TEM (c, d) of MWCNTs and O-MWCNTs

\section{Conclusion}

Orthogonal experiment analysis offered us an efficient and quickly method to determine the optimal oxidation process, and the optimal process is: $\mathrm{H}_{2} \mathrm{SO}_{4} / \mathrm{KMnO}_{4}$ (g/mg) 1:300, MWCNTs/KMnO 4 (g/g) 1:3, reaction temperature $150^{\circ} \mathrm{C}$, reaction time 7h. FT-IR, TGA and SEM, TEM measurement were taken follow by the optimal process. The result indicate that carboxyl groups were introduced to the surface of MWCNTs, structure of MWCNTs were damaged and MWCNTs became homogeneous dispersed.

\section{Acknowledgement}

The authors would like to acknowledge the research center for analysis \& measurement of Donghua University. This work is supported by Fundamental Research Program of Science and Technology Commission of Shanghai Municipality (13NM1401502).

\section{References}

[1] S. Iijima. Nature, 1991 (354) 56-58.

[2] Yizhe Wei, Xinglong Ling, Liming Zou, et al. Colloids and Surfaces A: Physicochemical and Engineering Aspects, 2015 (482) 507-513.

[3] Boshko Oleh, Dashevskyi, Mykola, Mykhaliuk, Olga, et al. Nanoscale research letters, 2016 (11) 78. 
[4] Hwang, Sheng-Jye; Chuang, Yu-Siang. Journal of alloys and compounds, 2016 (664) 284-290.

[5] Wang Qilong, Li Xiangkun, Di Yusong. Journal of applied physics, 2016 (119) 084504.

[6] Wen Liewei, Ding Wenzheng, Yang Sihua, et al. Biomaterials, 2016 (75) 163-173.

[7] Sergio Morales Torres, Tania L Silva, Luisa M Pastrana, et al. Phys. Chem. Chem. Phys, 2014 (16) 12237-12250.

[8] Xinlong Ling, Yizhe Wei, Liming Zou, et al. Applied Surface Science. 2013 (276) 159-166. 